\title{
La « laïcité » : entre la liberté d'expression et la liberté de croyance
}

I. En France, depuis les « caricatures de Mahomet » et les attentats de janvier et de novembre 2015, un débat public s'est amplifié, et les discussions ont mis en conflit les libertés d'expression et de croyance. Plusieurs questions ont inquiété, elles alarment toujours : la liberté d'expression peut-elle être absolue et illimitée ? Est-il acceptable de se moquer des religions ? Et s'il est acceptable de se moquer des croyances, peut-on alors se moquer de tout pour ne pas rompre avec le principe d'égalité ? Autre interrogation : y a-t-il -dans un contexte de laïcité- place pour un droit de l'homme au blasphème ? Ou au contraire le blasphème peut-il constituer une limite à la liberté d'expression ? Des réponses très diverses et souvent contradictoires ont été données à travers des disputes d'opinions et par le biais de la jurisprudence ${ }^{1}$. Or cet embarras autour de la liberté d'expression et de la liberté religieuse est augmenté par les remous qui bousculent depuis longtemps le concept de laïcité pourtant clairement défini par la doctrine juridique française comme la neutralité de l'État et la liberté de pratiquer une religion ou de ne pas pratiquer de religion, cette définition qui venait rendre à chacun le sien, dans le respect du principe des autonomies. On se rappelle -en 1949- de la conférence du professeur Jean Rivero, intitulée « La notion juridique de laïcité » qui a été publiée une seconde fois en 2005 dans le Recueil des archives de philosophie du droit consacré à la

1 Liberté d'expression et liberté de communication, Les nouveaux cahiers du Conseil constitutionnel, Paris, éd. Dalloz, 2012, n³6 ; Lä̈cité et liberté religieuse. Recueil de textes et de jurisprudence, Paris, Les éditions des journaux officiels, 2011 ; La laïcité, archives de philosophie du droit, Paris, éd Dalloz, mars 2005, t. 48 ; Le pluralisme, archives de philosophie du droit, Paris, éd Dalloz, mars 2006, t. 49 ; Philippe Chiappini, Le droit et le sacré, Paris, éd. Dalloz, 2006 ; Claude Durand-Prinborgne, La laïcité, éd. Dalloz, 2004 ; Dominique Laszlo-Fenouillet, La conscience, Paris, LGDJ, 1993 ; Convictions philosophiques et religieuses et droits positifs, colloque national de Moncton, Bruxelles, éd. Bruylant, 2010. 
Laïcité2. L'article situait bien la notion entre neutralité pour l'État et liberté de croyance. Toutefois les incertitudes autour de ce concept juridique qui semblait pourtant assez accordé, ont recommencé après les élections présidentielles de 1981. Ce fut alors le grand retour de la question laïque, au moment des célébrations hautement symboliques du centenaire des lois Ferry de 1881 et 1882. La commémoration prévue par le ministère de l'éducation nationale fut alors présentée dans une lettre au titre révélateur des mouvements qui vont suivre : «Fêtes du centenaire, fêtes de l'avenir $»^{3}$. C'était annoncer le réveil des discussions plus ou moins arrêtées depuis les lendemains de la seconde guerre mondiale. D'ailleurs, cette promulgation des lois laïques avait déjà été fêtée, avant la guerre, en 1931 : avant le centenaire, il y eut les journées du Cinquantenaire des lois Ferry, animées toujours par ce même souci de reprendre la définition du concept, et de le relier à la notion d'assimilation. On se souvient qu'en même temps se préparait l'exposition coloniale. De nombreuses revues -en 1931- avaient donc consacré des rubriques aux célébrations. Il s'agit entre autres de La Révolution prolétarienne fondée par Pierre Monatte, l'une des grandes figures du syndicalisme révolutionnaire. On y trouve un article d'Antoine Richard qui milita également pour le mouvement syndical. Le titre de son papier est là encore significatif, il marque la déception : « La mystique de 1881 et la réalité de $1931 »^{4}$. En voici un extrait qui montre toutefois combien le climat reste singulièrement enflammé : « Les débats parlementaires préparatoires du cinquantenaire, explique Antoine Richard, furent particulièrement passionnés. La Chambre dut tenir une séance de nuit fertile en apostrophes et invectives et qui faillit tourner en séance de pugilat $\rangle^{5}$, et le rédacteur de l'article de commenter « Curieuse préface pour une journée de prétendue concorde nationale $»^{6}$. Au moment des célébrations du centenaire, c'est-à-dire avec les millésimes 1980, cette effervescence resurgit. Mais cette fois, la carte religieuse de la France a changé, et l'on trouve donc ce même lien entre immigration, laïcité et convergence. C'est d'ailleurs 1'allocution de François Mitterrand du

2 Jean Rivero, « La notion juridique de laïcité », La laïcité, archives de philosophie du droit, op. cit., p. 257.

3 Bruno Poucet, « Grève et laïcité en France au début des années 1980 », Éducation et sociétés 2, 2007, n 20 , p. 31-45, URL : www.cairn.info/revue-education-et-societes-2007-2-page-31.htm.

4 Antoine Richard, «La mystique de 1881 et la réalité de 1931 », La Révolution prolétarienne, $\mathrm{n}^{\circ} 117,1931$ : « Nous devons tout d'abord énumérer ces lois laïques, charte de notre école primaire et même, en partie, des autres ordres d'enseignement. Jules Ferry fut ministre de l'Instruction publique de 1379 à 1883, avec une courte interruption et président du Conseil avec le même portefeuille du 23 septembre 1880 au 16 novembre 1881 et du 21 février au 20 novembre 1883, puis comme ministre des Affaires étrangères du 20 novembre 1883 au 30 mars 1885. C'est sous son administration directe que furent votées les lois scolaires dont l'ensemble est cohérent et animé du même esprit », p. 8-186.

5 Antoine Richard, « La mystique de 1881 et la réalité de 1931 », La Révolution prolétarienne, op. cit., p. 7-185.

6 Ibid. 
18 mai 1987 à l'occasion du colloque sur La pluralité des cultures, avec cette phrase que François Mitterrand qualifia lui-même d'imprudente : « c'est celle-là qui sera épinglée », ajoute-t-il. Voici la phrase : « Nous sommes français, nos ancêtres les gaulois, un peu romains, un peu germains, un peu juifs, un peu italiens, un petit peu espagnols, de plus en plus portugais, peut-être qui sait polonais, et je me demande si déjà nous ne sommes pas un peu arabes ? ${ }^{7}$ Ici l'on comprend -avec l'orientation du discours- la nouveauté de la situation qui met face à face les diversités culturelles, et surtout qui sous-entend des traditions religieuses différentes, l'une marquée par la distinction du politique et du religieux, l'autre qui au contraire associe le politique au religieux. D'un côté, il y a le «Rendez à César ce qui est à César et à Dieu ce qui est à Dieu » qui a été parfois entravé par le gallicanisme ; de l'autre, l'impossibilité de séparer. Mohamed Charfi qui avait été doyen de la faculté de droit de Tunis et ministre de l'éducation nationale, affirmait cette impossibilité dans son ouvrage Islam et liberté : « si la laïcité est la séparation entre l'Église et l'État, elle devient pour les sunnites impraticable, car ce serait la séparation entre l'État et le néant. La laïcité à la française s'adapte mal à l'Islam sunnite qui considère le service religieux comme un service public ; la nature de l'islam est telle que l'État ne peut se soustraire à ses obligations religieuses, de désigner les imams qui dirigent la prière à la mosquée, les professeurs qui enseignent dans les universités de théologie et les muftis qui conseillent les croyants sur les questions religieuses $\|^{8}$. Cette juxtaposition de traditions différentes interroge le socle de la laïcité devenu constitutionnel et qui -pour l'actualité la plus fraiche- vient d'être regardé par le pape François comme « exagéré et héritage de la Révolution française »'. C'est l'un des thèmes abordés par le souverain pontife dans son entretien accordé au journal La Croix, du 16 mai 2016. Puis au beau milieu de ces controverses, on voit surtout la forte tension entre les libertés d'expression et de religion qui désormais s'accentue, comme si les deux ordres normatifs n'étaient plus compatibles, alors que -dans la tradition chrétienne- César et Dieu doivent se distinguer et procéder à une juste répartition pour être en relation de réciprocité, ce sont les termes de l'Encyclique Deus caristas est ${ }^{10}$.

${ }^{7}$ Allocution de M. François Mitterrand, Président de la République, à l'occasion du colloque La pluralité des cultures organisé par la Fondation France-Libertés à La Sorbonne, Paris, 18 mai 1987.

${ }^{8}$ Mohamed Charfi, Islam et liberté, le malentendu historique, Paris, 1998, p. 193.

9 Entretien avec le Pape François, 16 mai 2016, La Croix : « En somme, voilà ce qui me fascine avec la France. D’un côté, cette laïcité exagérée, l'héritage de la Révolution française et, de l'autre, tant de grands saints "».

${ }^{10}$ Lettre encyclique Deus caristas est, Benoît XVI aux évêques, aux prêtres et aux diacres, aux personnes consacrées et à tous les fidèles laïcs sur l'amour chrétien, Paris, Pierre Téqui, 2006. 
II. Le retour de la question laïque. L'article de Jean Rivero commençait par une formule souvent reprise pour sa charge de provocation engagé contre le vocabulaire de la séparation : « la laïcité, disait-il : le mot sent la poudre, il éveille des résonances passionnelles contradictoires, et Jean Rivero poursuivait : cette contradiction n'est pas seulement celle, normale, qui oppose les esprits pour ou contre une notion claire; elle porte sur le contenu même de la notion, et le sens du mot $\gg{ }^{11}$. De la part du professeur de droit public, c'était exposer le tumulte à la fois des mots et des sens lorsqu'ils sont issus de la libre discussion pour la réforme de l'ordre juridique. On aperçoit alors l'un des mouvements de la formation du droit qui va des idées vers la règle. Mais il existe d'autres façons de fabriquer le droit, celle plus traditionnelle de former le droit positif à partir du droit naturel, des usages ou des nécessités. Ici pour l'élaboration du principe juridique de laïcité, on reste donc en pleine modernité, viennent d'abord les effervescences liées aux querelles d'opinions, et de ces discussions parfois houleuses découle la règle de droit. C'est toute l'histoire de la loi du 9 décembre 1905 qui certes avait été préparée par une succession de séparations partielles, mais dont la formulation sera surtout orientée par des prises de parole, par des idées qui font leur chemin et finissent souvent par s'imposer; on songe au discours d'Aristide Briand prononcé lors de la séance du 3 juillet 1905, juste avant le vote de la loi de séparation. Voici ce que -sur le processus d'adoption de la loi de séparation- disait René Rémond, en 2005, au moment du colloque de Sorèze : « On a vu quel avait été le rôle des personnalités, il était alors possible, par la puissance du verbe ou par la pertinence de l'argumentation d'infléchir le cours du débat parlementaire et de modifier le vote d'une partie des députés ${ }^{12}{ }^{2}$. C'est l'art du discours qui s'introduit dans le domaine du droit. Or habituellement les deux domaines, celui de la tribune et celui du droit, sont distincts et devraient toujours se distinguer. Il s'agit de deux mondes certes essentiels mais qui n'obéissent pas aux mêmes méthodes et ne recherchent pas la même finalité. Le monde des idées s'autorise en Occident la pluralité des significations. Depuis la Renaissance et les Lumières, c'est le jeu habituel des opinions qui s'entrechoquent, c'est aussi la règle de la langue ordinaire marquée par la polysémie alors que normalement l'art juridique appelle des définitions certaines et stables : selon la tradition romaine, le droit donne aux mots un sens et ne donne aux mots qu'un seul sens. Surtout, le droit ne doit jamais trahir sa finalité objective qui n'est pas celle des discussions, mais plutôt celle du juste dans le sens aristotélicien du terme, le droit ne pense pas la société au risque d'une dérive idéologique, celle de la logocratie -c'est-à-dire le règne du discours- qui ont rempli le $\mathrm{XX}^{\mathrm{e}}$ siècle. La difficulté

11 Jean Rivero, « La notion juridique de laïcité », La laïcité, archives de philosophie du droit, op. cit., p. 257.

12 René Rémond, «Conclusion », colloque de Sorèze, Regards croisés en 1905 sur la loi de séparation des Églises et de l'État, Presse du centre universitaire Champollion, 2005, p. 304. 
est donc d'envergure, elle rassemble ici deux domaines, puisque les débats fragilisent toujours les concepts juridiques rendus dès lors fort incertains aux yeux de l'opinion. C'est le cas pour la laïcité.

On s'en souvient, très tôt dès l'invention du mot lors des millésimes $1870^{13}$, la laïcité a été chahutée par plusieurs contraires parfois anti-religieux, et elle reste toujours maintenant plus ou moins encombrée par des épithètes qui l'ont qualifié de gardienne des libertés religieuses ou au contraire de facteur d'émancipation de ces mêmes libertés. Dès les années 1980 qui constituent une étape très importante pour cette notion, on a entendu parlé de laïcité positive ou négative, vraie ou falsifiée, fermée ou ouverte, rigide ou souple, de laïcité de dialogue ou de combat, on arrivait même à parler d'une laïcité laïque ou au contraire idéologique et spirituelle. La laïcité reçut également l'épithète de mystique : au moment du Cinquantenaire des lois Ferry, animé toujours par ce même souci de reprendre la définition du concept, Antoine Richard parle de la mystique de la laïcité et il insiste en disant : "Mystique, avons-nous dit, et le mot n'est pas déplacé, c'est la partie positive de cette souvre de laïcité. Laïcisons la morale et la philosophie comme nous avons laïcisé l'État, avec l'instituteur prêtre de la laïcité $\rangle^{14}$. Depuis la laïcité a également été entrevue comme ce principe extraordinaire qui unit les différences, et c'est l'une des dernières définitions que l'on peut répertorier depuis janvier 2016. Il s'agit ici d'une résurgence contemporaine de la coüncidentia oppositorum retrouvée par Nicolas de Cuse au moment de la chute de Constantinople et qui par la suite passionnera les milieux gnostiques. Avec cette série d'adjectifs et d'éparpillements, l'opinion assiste donc à la manifestation spectaculaire du syndrome babélien ou post-babélien. Il semble alors rendu impossible de concevoir une définition objective de ce principe, qui paraît de plus en plus insaisissable. Émile Poulat qui fut l'un des spécialistes de la laïcité et qui a beaucoup publié dans Politica hermetica, signalait la nature peut-être cachée ou énigmatique du terme : "la laïcité, personne ne sait ce dont on parle $»^{15}$. Puis le 31 décembre dernier, au moment de la parution du guide pratique, La laïcité au quotidien ${ }^{16}$, Régis Debray -dans un style assez amusé- déplorait lui aussi la confusion qui se maintient dans le discours :

13 « C'est en 1871 que le mot « laïcité » apparaît : le lexicographe Émile Littré le recense dans une citation tirée du journal La Patrie. Il faut toutefois attendre 1878 pour que le concept soit véritablement forgé et introduit par le philosophe de l'éducation Ferdinand Buisson dans son Dictionnaire de la pédagogie, considéré comme la «bible » de l'école laïque », Philippe Portier, « Aux sources de la laïcité », CNRS, le journal, 2015.

${ }^{14}$ Antoine Richard, "La mystique de 1881 et la réalité de 1931 », La Révolution prolétarienne, op. cit., p. 7-185.

15 Émile Poulat, « Le code de la laïcité est insatisfaisant », La Croix, 1 novembre 2011 ; « Enquête sur la laïcité perdue », Nouvelle revue Certitudes, avril, mai, juin 2003, nº14. 2015.

${ }^{16}$ Régis Debray, Didier Leschi, La laïcité au quotidien, Guide pratique, Gallimard, Folio, 
« la laïcité, dira-t-il, c'est une sorte de totem, de grigri que tout le monde invoque sur un mode incantatoire ${ }^{17}$. Pour la France, cette dispersion et ces ambiguïtés peuvent signifier une ignorance de la consistance du fait laïque, ce qui parait gênant, chacun donne son avis et continue encore à donner son avis. Comment alors expliquer l'attachement de l'opinion à la laïcité lorsque la laïcité elle-même est ignorée dans ses origines et sa nature, lorsque sa définition est rendue très peu sûre ? Aujourd'hui, on ne peut pas dire qu'il s'agit de vieilles polémiques qui n'ont plus cours, car il existe toujours des lieux où la querelle n'a pas cessé. Toutefois face aux bouleversements des idées, un aspect peut rassurer, c'est de voir combien ces bouillonnements et ces batailles ont leur utilité, ils préservent un espace pour les libertés et la forme démocratique. En revanche, une définition fixée pourrait être plus contraignante. On se souvient de l'ouvrage du poète polonais Czesław Miłosz publié à Paris, en 1953, La pensée captive, Essai sur les logocraties populaires $^{18}$; contre la pensée unique ou contre la nouvelle foi séculière du matérialisme dialectique, on pouvait y lire cette remarque à appliquer peutêtre aux situations françaises : les Occidentaux comparent « la démocratie à un radeau où règne le désordre, et où chacun rame dans une direction différente ». Tout le monde crie, s'injurie, et il n'est pas facile de se mettre d'accord sur la route à suivre. Miłosz qui recevra le Prix Nobel de Littérature en octobre 1980, au moment des grèves qui s'étendaient partout en Pologne, ajoute alors : « En comparaison au radeau, la rapide galère de la dictature qui file à force d'avirons, est un spectacle imposant. Il arrive pourtant parfois que le pauvre radeau flotte alors que la galère fait naufrage $»^{19}$.

Les débats autour de la laïcité font beaucoup penser à ce radeau où l'on se dispute beaucoup. Et c'est plutôt bon signe. Toutefois dans ces disputes habituelles, doivent être posées des limites à ne jamais franchir, celle de la haine et le terme a été utilisé par le législateur de juillet 1972, celle de la violence des pensées comme des actes. Nous le savons bien, tout commence par la pensée et par le verbe, par une hégémonie de l'esprit. Or la violence s'est introduite dans l'une des batailles que suscite le principe de laïcité, à savoir celle qui oppose les partisans de la liberté d'expression à ceux qui plutôt défendent la liberté de croyance sans être forcément des adversaires de la liberté d'expression, et vice versa. Il faut bien noter que les affrontements ne sont pas aussi simples. Ce ne sont pas seulement deux systèmes philosophiques qui se heurtent; les concurrences sont bien

17 « Régis Debray : la laïcité, c’est une sorte de totem », France Inter, émission du 30 décembre 2015.

18 Czeslaw Miłosz, La pensée captive, Essai sur les logocraties populaires, (Zniewolony umyst), traduit du polonais par A. Prudhommeaux et l'auteur, préface de Karl Jaspers, Paris, Gallimard, 1953, p. 58.

${ }^{19}$ Czeslaw Miłosz, La pensée captive, op. cit., p. 58. 
plus compliquées, car les systèmes qui s’affrontent ne relèvent pas de la même catégorie.

III. Le face à face entre liberté d'expression et liberté de croyance. Cettes questions qui aujourd'hui préoccupent beaucoup sur les libertés, et en particulier autour des insultes à caractère religieux, expriment un changement dans la façon de voir le lien entre Dieu et les hommes, ce changement qui a situé l'homme au centre de l'univers, à la place de Dieu, c'est l'article « encyclopédie », de l'Encyclopédie de Diderot. Mais l'histoire montre qu'il ne s'agit pas d'une crise récente, les discussions ont traversé les siècles ; elles réapparaissent toujours en temps de dislocations. Parmi les périodes d'intenses débats, il y eut le $\mathrm{XVI}^{\mathrm{e}}$ siècle et la redécouverte de l'antiquité païenne, avec le nouvel idéal de libertés garanties par l'État, avec l'émergence de nouvelles formes de blasphème donné par le libertinage. On y voyait un défi lancé par l'homme à Dieu, par le verbe humain qui entendait atteindre et blesser le Verbe de Dieu.

Auparavant, la glose médiévale voyait dans le blasphème une infraction mixte $^{20}$. Les docteurs de la foi disaient que c'était « un péché de la parole ou de la langue, de la langue de l'enfer $\|^{21}$; ce sont les travaux de Corinne Leveleux, La parole interdite. Le blasphème dans la France médiévale ${ }^{22}$. À propos du blasphème, Thomas d'Aquin donne une vision claire, on y voit nettement la distinction entre Dieu et l'homme, le passage de la Somme est très connu pour cette comparaison posée entre ce qui peut affecter l'homme et Dieu : « Si l'on compare entre eux l'homicide et le blasphème d'après les objets que visent ces péchés, il est évident que le blasphème, péché commis directement contre Dieu, l'emporte sur l'homicide, péché commis contre le prochain. Mais, si on les compare d'après la nocivité qu'ils produisent, l'homicide a la prépondérance, car il fait plus de mal au prochain que le blasphème n'en fait à Dieu $»^{23}$. Par la suite, pour l'Église catholique, la tendance médiévale à distinguer, à agir prudemment, à n'appliquer le blasphème qu'aux fidèles se maintiendra. On trouve cette attitude bien installée au XVIII ${ }^{\mathrm{e}}$ siècle dans le Dictionnaire de Trévoux ${ }^{24}$, puis au $\mathrm{XIX}^{\mathrm{e}}$ siècle et dans les codes de droit cano-

20 Brigitte Basdevant-Gaudemet, « Le blasphème, législation canonique et séculière, des Temps modernes au code de 1983 », Le blasphème du crime au péché, édité par Alain Dierkens et Jean-Philippe Schreiber, Bruxelles, éditions de l'université de Bruxelles, collection Problèmes d'histoire des religions, 2011, tome XXI, p. 95 à 106.

21 Ibid.

22 Corinne Leveleux, La parole interdite. Le blasphème dans la France médiévale (XIII -XVI siècles) : Du péché au crime, Paris, De Boccard, collection Romanité et Modernité du Droit, 2001.

23 Thomas d'Aquin, Somme théologique, secunda secundae, question 13 : Le péché de blasphème en général, article 3 : Le blasphème est-il le plus grand des péché ? Édition numérique : bibliothèque de l'édition du Cerf, 1999

24 Article « blasphème », Dictionnaire universel françois et latin contenant la signification et la définition tant des mots de l'une et l'autre langue, avec leurs différents usages, que des termes 
nique de 1917 et de 1983 : sont considérés comme blasphémateurs seulement les chrétiens qui offensent leur propre Dieu. Cet argument est d'importance, il pourrait d'ailleurs apporter des éléments pour servir à la réflexion et résoudre de nombreux accrochages.

Après les docteurs de la foi, viennent les docteurs de la loi. Dans un air très tôt chargé de gallicanisme, les juristes ont décrit le blasphème comme un crime d'une gravité évidente. Avec le triomphe de l'État, le blasphème devait devenir un crime de lèse-majesté que les parlements ont puni plus sévèrement que les juridictions ecclésiastiques. Durant le second XVIII ${ }^{e}$ siècle, lorsque la sanction de ces crimes se fait plus rare, on écrira beaucoup en faveur du blasphème ${ }^{25}$. En France, avec la modernité et contre la catholicité, se forme dès lors une forte tradition blasphématoire. Les penseurs des Lumières défendront cette liberté absolue d'expression que la Déclaration des droits de l'homme et du citoyen du 26 août 1789 n'introduira pas, puisque les articles 4, 10 et 11 exigent les limites de la loi pour éviter les abus. L'équilibre juridique semblait déjà difficile à établir entre la liberté d'expression réclamée par la République des lettres et les libertés religieuses qui rapidement ne coïncident plus. Et contre la religion, souvent l'on a redonné la formule de Saint-Just : pas de liberté pour les ennemis de la liberté. La Révolution venait tout à la fois durcir l'affrontement entre la liberté d'expression et la liberté de croyance, et aussi aplatir les opinions religieuses, auparavant considérées comme supérieure, parce qu'il en va de la relation à Dieu. Et c'est l'article 10 de la Déclaration qui assure ce nivellement :« Nul ne doit être inquiété pour ses opinions, même religieuses, pourvu que leur manifestation ne trouble pas l'ordre public établi par la Loi $»^{26}$.

Avec l'époque contemporaine, cette difficile confrontation semble plutôt servir la liberté d'expression. Les satires religieuses sont devenues -à partir de 1984- plus nombreuses, elles ont abouti à des procédures judiciaires. Les poursuites pénales se sont succédées contre le rédacteur en chef de Charlie Hebdo. C'est aussi la sortie de films contestés par une partie de l'opinion,

propres de chaque état et de chaque profession, ou Dictionnaire de Trévoux, imprimé à Nancy en 1740, chez Pierre Antoine.

${ }_{25}$ Jacqueline Hoareau-Dodinau, Dieu et le Roi. La répression du blasphème et de l'injure au roi à la fin du Moyen Âge, Cahier de l'Institut d'anthropologie juridique, Presses universitaires de Limoges, Limoges, 2002.

${ }^{26}$ Article $4:$ : La liberté consiste à pouvoir faire tout ce qui ne nuit pas à autrui : ainsi, l'exercice des droits naturels de chaque homme n'a de bornes que celles qui assurent aux autres Membres de la Société la jouissance de ces mêmes droits. Ces bornes ne peuvent être déterminées que par la Loi », article 10: «Nul ne doit être inquiété pour ses opinions, même religieuses, pourvu que leur manifestation ne trouble pas l'ordre public établi par la Loi», article $11:$ : La libre communication des pensées et des opinions est un des droits les plus précieux de l'Homme : tout Citoyen peut donc parler, écrire, imprimer librement, sauf à répondre de l'abus de cette liberté dans les cas déterminés par la Loi », Déclaration des droits de l'homme et du citoyen, 26 août 1789, site Légifrance. 
comme La dernière tentation $d u$ Christ, de Martin Scorsese, la Cour de Cassation précisera en octobre 1990 que "sont de valeur égale le principe de liberté d'expression notamment en matière artistique et le principe lié au respect dû aux croyances de Martin Scorsese $»^{27}$. Puis il y aura le film Je vous salue Marie de Jean-Luc Godard, avec un procès perdu par les plaignants ${ }^{28}$; ou la photographie controversée Piss Christ, ainsi que la pièce présentée au festival d'Avignon, Sur le concept du visage du fils de Dieu de Romeo Castellucci, puis ce fut la programmation de Golgotha Picnic. Ces productions ont déclenché des débats qui ont profondément divisé l'opinion, des plaintes ont été déposées, mais elles n'ont pas abouti ${ }^{29}$.

Il faut aussi rappeler le procès de Michel Houelebecq engagé contre ses propos sur l'islam. Puis parmi les derniers troubles en France, on peut penser aux procès contre les militantes femens en 2013, qui n'ont pas été condamnées.

Ces affaires furent l'occasion de discussions sur le concept de la liberté d'expression contre la liberté de croyance, avec les interventions des plus célèbres polémistes qui ont proclamé une liberté absolue et illimitée d'expression. Selon la controverse et comme le blasphème ne pouvait servir de limite à la liberté d'expression, on livre alors la maxime : la liberté ne peut être partagée, elle est irréductible, avec comme postulat, le principe, c'est la liberté;

27 Jean Boulègue, Le blasphème en procès, 1984-2009, L'église et la mosquée contre les libertés, Nova Éditions, 2010 ; Cour de cassation, chambre civile 1, audience publique du 29 octobre 1990, $\mathrm{n}^{\circ}$ de pourvoi : 88-19366, Bulletin 1990 I, n 226 p. 161 : « Mais attendu que le principe de la liberté d'expression, notamment en matière de création artistique, d'une part, comme, d'autre part, celui du respect dû aux croyances et le droit de pratiquer sa religion étant d'égale valeur, il appartenait aux juges du fait de décider des mesures appropriées à faire respecter ce nécessaire équilibre ; que sans nier la possibilité d'abus de droit en de pareils domaines, qui constitueraient alors des troubles manifestement illicites, la cour d'appel, -qui a relevé qu'il y avait lieu d'éviter « que quiconque se trouve, parce que non prévenu, en situation d'être atteint dans ses convictions profondes » au même titre que de ne pas porter atteinte à la liberté d'expression- a pu estimer qu'il n'y avait pas eu, en la circonstance, trouble manifestement illicite et décider souverainement des mesures les plus appropriées à préserver le juste équilibre des droits et libertés en cause ; que, ce faisant, elle n'a pas pris en considération la subjectivité personnelle de tel ou tel spectateur et qu'elle a répondu, par la généralité des mesures prises, aux préoccupations exprimées dans les conclusions invoquées ; que le moyen ne peut être accueilli en aucune de ses branches ».

28 Jean Boulègue, Le blasphème en procès, 1984-2009, L'église et la mosquée contre les libertés, op. cit. ; Cour de cassation, chambre civile 1, audience publique du mardi 21 juillet 1987, $\mathrm{n}^{\circ}$ de pourvoi : 85-15044, Bulletin $1987 \mathrm{I}, \mathrm{n}^{\circ} 236$, p. 172 : « attendu qu'après avoir fait sien le motif de l'ordonnance critiquée devant elle, motif suivant lequel l'auteur d'une oeuvre de l'esprit doit bénéficier du droit à la liberté d'expression, sans autres restrictions que celles imposées par la loi dès lors que, dans sa finalité ou son expression, l'oeuvre ne constitue pas une apologie de crimes ou délits sanctionnés par la loi pénale ou une provocation à en commettre, la cour d'appel, qui retient que le trouble créé par le film litigieux résidait dans le caractère blessant qu'il présentait pour diverses catégories de personnes, a, par cette appréciation de fait, exclu le délit de provocation à la discrimination, à la haine et à la violence et répondu aux conclusions ».

29 Ibid. 
la restriction, c'est l'exception ${ }^{30}$. Il s'agit également de la campagne lancée par Reporters sans frontières (RSF), après les attentats de janvier 2015 qui a été très peu suivie, puisque la déclaration alors proposée sur la liberté d'expression n'a été signée que par une trentaine de dignitaires religieux sur les 10000 signatures attendues, ce qui peut signifier -en période de tumultes- la lassitude de la société française. Très rapidement, les débats sont passés de l'infinie liberté d'expression à la proclamation du droit absolu de blasphémer, parfois confondu avec le droit à l'humour, blasphème et humour ne se distinguaient plus, or personne ne gagne à parler un un langage confus. Puis, en janvier 2015, après les attentats, un élément nouveau est apparu, c'est la sacralisation du droit de blasphémer avec la formule souvent utilisée « notre droit sacré au blasphème qui doit être garanti » ou encore autre slogan «Le droit au blasphème, notre bien le plus sacré ». Ici l'on peut saisir le renversement qui s'opère et qui peut transforme la liberté absolue d'expression en négation de la liberté religieuse.

En plus des discussions, des livres ont également été écrits, dès 1989 paraît Le droit de blasphémer d'Orlando de Rudder ${ }^{31}$, qui justifie -sans craindre le mélange des deux sphères- les insultes à caractère religieux comme fondement de la démocratie. Depuis 2015, le blasphème est aussi devenu le thème central de nombreux colloques et essais, et cette abondance affiche la sensibilité du sujet : une rencontre s'est tenue à Poitiers, Le blasphème dans une société démocratique ${ }^{32}$. Puis furent publiés L'éloge du blasphème de Caroline Fourest $^{33}$, ainsi que de plusieurs histoires du blasphème, par exemple celles d'Alain Cabantous, de Jacques de Saint-Victor ${ }^{34}$.

À travers cette abondance des déclarations et des écrits, un questionnement surgit, celui des limites juridiques de la liberté d'expression qui ont été reconnues par l'article 4 de la Déclaration de 1789, puis précisées par la Loi sur la liberté de la presse du 29 juillet 1881. Vient ensuite la loi Pleven du 1er juillet 1972 qui a créé, par son article $1^{\text {er }}$, un nouveau délit « d'incitation à la discrimination, à la haine ou la violence à l'égard d'une personne ou un groupe de personnes à raison de leur origine ou de leur appartenance ou leur

${ }^{30}$ La formule a été donnée par l'arrêt Baldy, Conseil d'État, 10 août 1917, « La liberté est la règle, larestrictionde policel'exception », elle est maintenant simplifiée et élargie à des conflits autres que ceux d'ordre public visés par la jurisprudence du Conseil d'État.

31 Orlando de Rudder, Le droit de blasphémer, Paris, Renaudot, 1989.

${ }^{32}$ Fabien Marchadier, Le blasphème dans une société démocratique, Dalloz-Thèmes et Commentaires, Paris, 2016.

33 Caroline Fourest, L'éloge du blasphème, Paris, Grasset, 2015, qui écrit : « Menacés par les fanatiques, censurés par les lâches, les esprits libres de tous les continents n'en finissent plus de se battre, sur tous les fronts, pour maintenir un monde éclairé. La lumière qui les guide s'appelle le droit au blasphème ».

${ }^{34}$ Alain Cabantous, Histoire du blasphème en Occident, Paris, Albin Michel, 2015 ; Jacques de Saint-Victor, Blasphème :brève histoire d'un crime imaginaire, Paris, Gallimard, 2016. 
non appartenance à une ethnie, une nation, une race ou une religion $»^{35}$. Ce délit pouvait être puni d'un mois à un an d'emprisonnement et d'une amende de 2000 à 300000 francs. Le texte de 1972 venait donc indiquer sur le plan juridique les retraits communautaires et les exclusions qui, en France, commençaient à s'amplifier sans reconnaître toutefois le principe de respect des religions. Dans la France contemporaine, la doctrine juridique explique que les droits de l'Homme visent à protéger les individus et non les systèmes de pensée. Une assimilation est donc faite entre religions et idée abstraite, auparavant distinguées. On comprend combien l'époque contemporaine s'est écartée de la tradition chrétienne pour aller vers un déisme que peut supporter l'incroyance.

Par ailleurs, une autre interrogation à la fois juridique et politique tracasse, on peut se demander aujourd'hui s'il n'est pas contradictoire de parler d'intégration des étrangers et de proclamer en même temps le droit de blasphémer. N'est-il pas étonnant de se moquer des croyances religieuses de ceux que les gouvernements veulent héberger sur le sol national et encourager les insultes à caractère religieux. Le droit au blasphème qui est un droit subjectif doit faire face au principe juridique de responsabilité. Pour les juristes, ce principe de responsabilité est le fondement du droit objectif, c'est un principe de justice, qui trouve son origine dans la maxime de droit romain justicia praecipit suum cuique reddere. Par conséquent, la liberté est un droit subjectif qui ne peut se dissocier de la responsabilité. Liberté et responsabilité vont ensemble. Lorsque la violence aggrave les crises, la solution la plus facile et la plus séduisante -mais qui n'est sûrement pas la meilleure- est de dire ce qui est bon selon le législateur ${ }^{36}$. La loi devient donc souveraine maîtresse du bien et du mal, souveraine maîtresse des libertés, ce qui n'a jamais été le rôle de la loi, sauf lors des dérives idéologiques.

À propos del'article 2 de la Loi de 1905 sur la séparation des Églises et de l'État proclame que « la République ne reconnaît aucun culte» ${ }^{37}$. Toutefois et paradoxalement, la reconnaissance du droit au blasphème devient une négation de la loi de séparation, une négation de la laïcité. Si le blasphème n'existe pas comme infraction sous la loi française,si cette notion n'est pas qualifiée juridiquement,comment peut-il y avoir un droit au blasphème $?^{38}$ Contre la volonté parfois proposée de faire du blasphème un droit individuel, se trouve une mise en garde et une interrogation de bon sens, souvent renouvelée par les juristes, est-ce bien nécessaire ? Dans la société française, le blasphème est

35 Loi n $72-546$ du 1 juillet 1972 relative à la lutte contre le racisme, site Légifrance.

36 Jean-Marc Trigeaud, « Mœurs religieuses et laïcité ou la limite des lois non écrites », La laïcité, archives de philosophie du droit, op. cit., p. 57 à 73.

37 Article 2 de la Loi du 9 décembre 1905 concernant la séparation des Églises et de l'État.

38 Isabelle de Gaulmyn, «Le droit au blasphème, nouveau droit français », La Croix, 20 janvier 2016. 
une insulte d'ordre religieux, ce n'est pas une liberté d'ordre politique. Là encore, il ne faut pas négliger les distinctions, pour éviter le risque de soumettre le juridique à un ordre néo-moral et « de le placer sous la coupe de la science de l'individu dans la méconnaissance des fins du droit $\gg{ }^{39}$.

Pour saisir les enjeux de ces tumultes qui remplissent de plus en plus l'air occidental, peuvent être repris un essai de Michel Villey, Le droit et les droits de l'homme; on y trouve une réflexion sur l'usage des libertés d'expression et de croyances. Michel Villey y montrait combien la liberté d'expression n'est pas pour tous et dans l'intérêt de tous, mais pour un petit nombre, pour une élite alors que la liberté de croire ou de ne pas croire est pour tous ...or ce mouvement élitiste -explique le philosophe- représente une menace de décomposition, lorsque les libertés ne sont pas distribuées équitablement entre religions et État, entre Dieu et César ${ }^{40}$.

\section{BIBLIOGRAFIA}

Allocution de M. François Mitterrand, Président de la République, à l'occasion du colloque La pluralité des cultures organisé par la Fondation France-Libertés à La Sorbonne, Paris, 18 mai 1987.

Article 2 de la Loi du 9 décembre 1905 concernant la séparation des Églises et de l'État.

Basdevant-Gaudemet B., Le blasphème, législation canonique et séculière, des Temps modernes au code de 1983, w: "Le blasphème du crime au péché", tome XXI, 2011.

Boulègue J., Le blasphème en procès, 1984-2009, L'église et la mosquée contre les libertés, Nova Éditions, 2010.

Cabantous A., Histoire du blasphème en Occident, Paris, 2015.

Charfi M., Islam et liberté, le malentendu historique, Paris, 1998.

Chiappini P., Le droit et le sacré, Paris, éd. Dalloz, 2006.

Convictions philosophiques et religieuses et droits positifs, colloque national de Moncton, Bruxelles, éd. Bruylant, 2010.

Debray R., Leschi D., La laïcité au quotidien, Guide pratique, Gallimard, Folio, 2015.

Déclaration des droits de l'homme et du citoyen, 26 août 1789, site Légifrance.

Dictionnaire universel françois et latin contenant la signification et la définition tant des mots de l'une et l'autre langue, avec leurs différents usages, que des termes propres de chaque état et de chaque profession, ou Dictionnaire de Trévoux, imprimé à Nancy en 1740, chez Pierre Antoine.

Durand-Prinborgne C., La laïcité, éd. Dalloz, 2004.

39 Michel Villey, Le droit et les droits de l'homme, Paris, Presses universitaires de France, 1983 , p. 42.

40 Ibid. 
Entretien avec le Pape François, 16 mai 2016, w: "La Croix".

Fourest C., L'éloge du blasphème, Paris, Grasset, 2015.

Gaulmyn I. de, Le droit au blasphème, nouveau droit français, w: "La Croix", 20 janvier 2016.

Hoareau-Dodinau J.,Dieu et le Roi. La répression du blasphème et de l'injure au roi à la fin du Moyen Âge, Limoges, 2002.

Laïcité et liberté religieuse. Recueil de textes et de jurisprudence, Paris, Les éditions des journaux officiels, 2011.

Laszlo-Fenouillet D., La conscience, Paris, LGDJ, 1993.

Le pluralisme, archives de philosophie du droit, Paris, éd Dalloz, t. 49., mars 2006.

Lettre encyclique Deus caristas est, Benoît XVI aux évêques, aux prêtres et aux diacres, aux personnes consacrées et à tous les idèles laïcs sur l'amour chrétien, Paris, Pierre Téqui, 2006.

Leveleux C., La parole interdite. Le blasphème dans la France médiévale (XIII ${ }^{e}-X V I^{e}$ siècles): Du péché au crime, Paris, 2001.

Liberté d'expression et liberté de communication, w: "Les nouveaux cahiers du Conseil constitutionnel”, Paris, éd. Dalloz, n³6, 2012.

Loi $n^{\circ}$ 72-546 du 1 juillet 1972 relative à la lutte contre le racisme, site Légifrance.

Marchadier F., Le blasphème dans une société démocratique, Dalloz-Thèmes et Commentaires, Paris, 2016.

Miłosz Cz., La pensée captive, Essai sur les logocraties populaires, tłum. A. Prudhommeaux, Paris, 1953.

Poucet B., Grève et laïcité en France au début des années 1980, w: "Éducation et sociétés 2", no 20, 2007, URL : www.cairn.info/revue-education-et-societes-20072-page-31.htm.

Poulat É., Enquête sur la laïcité perdue, w: "Nouvelle revue Certitudes”, n¹4, avril, mai, juin 2003.

Poulat É., Le code de la laïcité est insatisfaisant, w: "La Croix", 1 novembre 2011 ;

Rémond R., Conclusion, colloque de Sorèze, Regards croisés en 1905 sur la loi de séparation des Églises et de l'État, Presse du centre universitaire Champollion, 2005.

Richard A., La mystique de 1881 et la réalité de 1931, w: "La Révolution prolétarienne", n' $117,1931$.

Rivero J., La notion juridique de laïcité, w: "La laïcité, archives de philosophie du droit", t. 48., Paris, mars 2005.

Rudder O. de, Le droit de blasphémer, Paris, 1989.

Saint-Victor J., Blasphème: brève histoire d'un crime imaginaire, Paris, 2016.

Thomas d'Aquin, Somme théologique, secunda secundae, 1999.

Trigeaud J.-M., Mśurs religieuses et laïcité ou la limite des lois non écrites, w: "La laïcité, archives de philosophie du droit", t. 48, Paris, mars 2005.

Villey M., Le droit et les droits de l'homme, Paris, 1983. 


\section{"LAÏCITÉ", POMIĘDZY WOLNOŚCIĄ WYZNANIA A WOLNOŚCIĄ SŁOWA}

\section{Streszczenie}

We Francji, od czasu „Komiksów o Mahomecie” oraz ataków w styczniu 2015 roku, szeroka debata publiczna przybrała na sile, a dyskusja wciągnęła w konflikt wolność wyznania z wolnością słowa. Po tych wydarzeniach ciągle zadawane są pytania: Czy robienie sobie żartów z religii jest do przyjęcia? Czy istnieje ludzkie prawo do bluźnierstwa? Czy wolność wyrażania religijnego i antyreligijnego jest absolutna i nieograniczona? Czy jest zatem konieczne rozróżnienie tych dwóch zestawów praw w celu uniknięcia konfliktu? Bardzo różne oraz czasem bardzo sprzeczne odpowiedzi pojawiają się w dyskusji, także za pomocą nauk prawnych. Ta różnorodność opinii dodaje przede wszystkim dwuznaczności, ponieważ prawa podstawowe wydają się sprzeczne z różnymi deklaracjami praw człowieka. We Francji, te kwestie pokazują głęboki i niebezpieczny kryzys w społeczeństwie, odmienny od tego z początku XX wieku, z czasu uchwalonego w 1905 roku prawa o Rozdziale Kościoła od Państwa.

W obliczu niepewności, ważnym jest pamiętanie o historii konfliktów we Francji pomiędzy wolnością wyznania i wolnością słowa, aby zrozumieć filozoficzną, religijną oraz polityczną podstawę tych wolności, aby zauważyć trudności w wyborze rozwiązań doktrynalnych i prawnych. Artykuł 10 Deklaracji Praw Człowieka i Obywatela zawiera i broni tych dwóch wolności, które są obecnie sprzeczne ze sobą: „Nikt nie może być niepokojony z powodu swoich opinii, nawet tych religijnych, pod warunkiem, że ich okazywanie nie powoduje problemów w ustanowionym przez prawo porządku publicznym”. Korzystanie z tych wolności jest ograniczone przez poszanowanie prawa, jest to ograniczenie prawne. A więc to podnosi kwestie nastawienia francuskiego ustawodawcy w kontekście silnej radykalizacji oraz stanu wyjątkowego.

Ponadto w dzisiejszej Francji, zakłopotanie nagromadzone wokół wolności słowa jest powiększone przez debaty na temat konceptu „laïcité”, który jest definiowany przez francuską doktrynę prawną jako „neutralność Państwa” i „wolność praktykowania religii oraz prawo do niepraktykowania religii”. Ale obecnie te debaty sprawiły, że definicje prawne stają się niepewne w oczach opinii publicznej, ponieważ kilka przymiotników opisuje sekularyzm: pozytywny i negatywny, otwarty i zamknięty, etc.

Te debaty wzmagają niepokoje, których francuska polityka integracyjna ma trudności z kontrolowaniem, aby utrzymać „,wspólne życie”. 


\section{THE "LAÏCITÉ": \\ BETWEEN FREEDOM OF BELIEF AND FREEDOM OF SPEECH}

\section{Summary}

In France, since the "Cartoons of Muhammad" and the attacks in January 2015, a wide public debate has amplified, and the discussions bring into conflict freedom of belief with freedom of speech. After these events, questions are repeatedly asked: Is it acceptable to make fun of religions? Is there a human right to blaspheme? Is freedom of religious or antireligious expression absolute and unlimited? Is it then necessary to distinguish two orders of rights to avoid conflicts? Very different and sometimes very contradictory responses are made in the discussion, and through the jurisprudence. This diversity of opinions adds ambiguity above all because fundamental rights appear conflicting in the various declarations of human rights. In France, these issues show a deep and dangerous crisis of the society, different from that of the early $20^{\text {th }}$ century, from the time of the Law of Separation of the Churches and State from 1905.

Faced with uncertainties, it should be important to remember the history of the French conflicts between freedom of religion and freedom of speech, to understand the philosophical, religious and political basis of those freedoms, to see the difficulty in choosing doctrinal and legal solutions. Article 10 of The Declaration of the Rights of Man and of the Citizen contains and protects these two freedoms that today are in opposition: "No one may be disturbed for his opinions, even religious ones, provided that their manifestation does not trouble the public order established by the law". The exercise of these freedoms is limited by the respect of law; it is a legal limit. So, this raises the question of the attitude of the French legislator in the context of violent radicalization and state of emergency.

Moreover, today in France, the embarrassment around freedom of speech is increased by debates on the concept of "laïcité" that the French legal doctrine defines as "the neutrality of the State" and "the freedom to practice religion or not to practice religion". But the debates have made this legal definition uncertain in the eyes of the public opinion because several adjectives describe secularism: positive or negative, open or closed, etc.

These debates exacerbate discords that the French integration policy has difficulties controlling in order to maintain the "living together". 